\title{
The Graduate Student Symposium of Diagrams 2010
}

\author{
Jim Davies \\ Institute of Cognitive Science, Carleton University \\ 1125 Colonel By Drive, Ottawa, Ontario, Canada K1S5B6 \\ jim@jimdavies.org
}

The Graduate Student Symposium (GSS) is a forum at which Ph.D. students engaged in diagrams research have an opportunity to present their research and interact with established scientists as well as other students.

The GSS has two main goals. First, the GSS provides an environment that is supportive of constructive feedback. Second, it is an opportunity for the students to shine, take the focus, and network with their peers and more senior scholars.

Several papers were submitted only to the GSS. There were papers based on formal analyses (Burton, Howse, Stapleton, \& Hamie; Delaney) modeling human understanding (Dickmann; Smuc) and visual language creation for architectural design (Hamadah).

The other presentations were based on students' papers presented in the Diagrams 2010 poster session. Nearly all of these papers involved human experimentation or modeling. Several papers dealt specifically with diagrams in education, others on basic understanding of diagrams, and others on diagram generation. One paper presented work that developed a visual language for mathematical proof.

In addition, two invited speakers gave presentations, one on how to present a paper at a conference, and another with dissertation advice.

A bursary for student attendees to the GSS (totaling $\$ 20,000$ USD) was generously provided by the National Science Foundation. All students who applied for funding received a bursary to help them pay for attending the conference.

In summary, the Graduate Student Symposium of Diagrams 2010 provides a unique opportunity for feedback to students involved with diagrams research. Further, the topics students choose gives us insight into the future of our field. 